\title{
Degradación Ruminal de Dietas a Base de Biomasa Pos-cosecha de Amaranthus cruentus: Efecto sobre los Protozoos del Rumen y Producción de Gas in vitro
}

\author{
Rumen Degradation of Diets Based on Post-harvest Biomass of Amaranthus \\ cruentus: Effect on Rumen Protozoa and in vitro Gas Production
}

\author{
M. Barros-Rodríguez ${ }^{1,3}$, J. Oña-Rodríguez ${ }^{1}$, R. Mera-Andrade ${ }^{1}$, J. Artieda-Rojas ${ }^{1}$, S. \\ Curay-Quispe $^{1}$, D. Avilés-Esquivel ${ }^{1}$, J. Solorio-Sánchez ${ }^{2}$, C. Guishca-Cunuhay ${ }^{1}$
}

\section{Resumen}

\begin{abstract}
El objetivo de esta investigación fue evaluar la cinética de degradación ruminal de los nutrientes in situ, así como la digestibilidad aparente de los nutrientes, población de protozoarios del rumen y producción de gas in vitro de dietas a base de biomasa poscosecha de Amaranthus cruentus. La biomasa pos-cosecha del $A$. cruentus con el forraje de Pennisetum clandestinum se mezcló en base seca de acuerdo a los siguientes tratamientos: T1: 100\% A. cruentus, T2: 100\% P. clandestinum, T3: 75\% P. clandestinum + $25 \%$ A. cruentus, T4: $50 \%$ P. clandestinum $+50 \%$ A. cruentus, bajo un diseño completamente aleatorizado. Los parámetros de degradación ruminal muestran diferencias entre tratamientos $(\mathrm{p}<0.05)$, obteniendo la mayor degradación efectiva de la materia seca (MS), proteína cruda (PC) y fibra detergente neutro (FDN) en T1 (423.4, 404.1 y $354.5 \mathrm{~g} / \mathrm{kg} \mathrm{MS}$, respectivamente). La digestibilidad in vitro de la $\mathrm{MS}$, materia orgánica (MO) y PC fue mayor ( $\mathrm{p}<0.05)$ en T1 $(60,68$ y 59\%, respectivamente) y T4 $(53,61$ y $51 \%$, respectivamente). La menor producción de gas in vitro fue para $\mathrm{T} 1$, con una diferencia aproximada de $91 \mathrm{ml} / 0.5 \mathrm{~g}$ MS fermentable con respecto a los demás tratamientos $(\mathrm{p}=0.0001)$. La menor $(\mathrm{p}=0.0026)$ población de protozoarios Holotricos y Entodiniomorfos se observó en T1 ( 0 y $1.80 \log _{10}$, respectivamente). Se concluye que la biomasa pos-cosecha de $A$. cruentus al incorporarlo a la dieta de los rumiantes puede incrementar la degradación y digestibilidad de los nutrientes y reducir la producción de gases de efecto invernadero y las poblaciones de protozoarios del rumen.
\end{abstract}

Palabras clave: Amaranthus cruentus; protozoarios; degradación ruminal; producción de gas in vitro

\footnotetext{
${ }^{1}$ Facultad de Ciencias Agropecuarias, Universidad Técnica de Ambato, Cevallos, Tungurahua, Ecuador

${ }^{2}$ Facultad de Medicina Veterinaria y Zootecnia, Universidad Autónoma de Yucatán, Mérida, Yucatán, México

${ }^{3}$ E-mail: ma_barrosr@yahoo
}

Proyecto financiado por la Universidad Técnica de Ambato, Ecuador, a través de la Dirección de Investigación y Desarrollo (DIDE). Resolución CU-0135-P-2014

Recibido: 24 de enero de 2017

Aceptado para publicación: 29 de mayo de 2017 
The aim of this research was to evaluate the in situ rumen degradation kinetics of nutrients, as well as the apparent digestibility of nutrients, population of rumen protozoa, and in vitro gas production of based diets from post-harvest biomass of Amaranthus cruentus. Post-harvest biomass of $A$. cruentus with Pennisetum clandestinum forage was mixed in dry basis according to four treatments: T1: $100 \%$ A. cruentus, T2: $100 \%$ P. clandestinum, T3: 75\% P. clandestinum $+25 \%$ A. cruentus, T4: $50 \%$ P. clandestinum + $50 \%$ A. cruentus, under a completely randomized design. The ruminal degradation parameters showed differences between treatments $(\mathrm{p}<0.05)$ obtaining the highest effective degradation of dry matter (DM), crude protein (CP) and neutral detergent fibre (NDF) in $\mathrm{T} 1(423.4,404.1$ and $354.5 \mathrm{~g} / \mathrm{kg}$, respectively). The in vitro digestibility of DM, organic matter $(\mathrm{OM})$ and $\mathrm{CP}$ was higher $(\mathrm{p}<0.05)$ in $\mathrm{T} 1(60,68$ and $59 \%$ respectively) and $\mathrm{T} 4$ (53, 61 and $51 \%$ respectively). The lowest in vitro gas production was for $\mathrm{T} 1$, with a difference of approximately $91 \mathrm{ml} / 0.5 \mathrm{mg}$ fermented DM with respect to the other treatments $(\mathrm{p}=0.0001)$. The lowest $(\mathrm{p}=0.0026)$ population of protozoa Holotrichs and Entodinomorphs was observed in T1 ( 0 and $1.80 \log _{10}$ respectively). It is concluded that the post-harvest biomass of $A$. cruentus when incorporated into the diet of ruminants, can increase the degradation and digestibility of nutrients, and reduce the production of greenhouse gases and populations of protozoa of the rumen.

Key words: Amaranthus cruentus; protozoa; rumen degradation; in vitro gas production

\section{INTRODUCCIÓN}

El desempeño productivo de los rumiantes está en función del valor nutricional de la dieta que consumen. Asimismo, la dieta es el factor más determinante sobre el tipo y las proporciones de poblaciones microbianas del rumen, así como su capacidad para degradar carbohidratos estructurales como la celulosa, hemicelulosa y compuestos tóxicos, por lo que determina el perfil de fermentación ruminal (Tan et al., 2011). En sistemas de producción en condiciones de los páramos del Ecuador, la principal fuente de alimentación la constituyen pastos y forrajes de calidad nutritiva deficiente (Manotoa, 2016), lo que puede ocasionar pérdidas de energía en forma de gases efecto invernadero $\left(\mathrm{CO}_{2}, \mathrm{CH}_{4}\right)$ del 8 al 12\%, debido a los altos niveles relativos de fibra y lignina presentes en la dieta (Kurihara et al., 1999).
Una alternativa para mejorar el rendimiento productivo en los rumiantes a través de la alimentación es el aprovechamiento de la biomasa pos-cosecha de los cultivos agrícolas del altiplano andino del Ecuador, ya que se producen altas cantidades de chocho chocho (Lupinus mutabilis), avena, (Avena sativa), quinoa (Chenopodium quinoa), amaranto (Amaranthus cruentus), etc. La incorporación de estas especies, ricas en nutrientes y compuestos secundarios, en la dieta de los rumiantes, se presenta como una alternativa en los páramos del país, con el fin de formular estrategias de manipulación de la fermentación ruminal, orientadas a mejorar la eficiencia en el rendimiento de los animales y reducir los gases de efecto invernadero (Abdulrazak et al., 2000). Del cultivo de amaranto se aprovecha la inflorescencia (panoja) para el consumo humano, quedando como residuo la planta con hojas, tallos y pequeñas panojas, donde los tallos y hojas po- 
seen niveles de proteína cruda (PC) de 9.5 y $24.8 \%$, respectivamente (García-Pereyra et al., 2009).

El amaranto es un forraje rico en proteína, lo que propicia mayor disponibilidad de compuestos como amoniaco, aminoácidos y péptidos, así como ácidos grasos de cadena corta ramificados, que se producen como resultado de la degradación de las proteínas (Tan et al., 2011). Estas sustancias favorecen la degradación de la fibra, actuando como activadores del crecimiento de bacterias ruminales, especialmente las celulolíticas y proteolíticas (Hoover y Stokes, 1991). El amaranto tiene, además, compuestos secundarios como los taninos (hidrolizables y condensados), que forman complejos con las proteínas proporcionando mayor proteína de sobrepaso hacia las partes bajas del tracto gastrointestinal, así como una posible acción directa de los compuestos secundarios (taninos, saponinas, ácidos orgánicos) que pueden reducir la actividad o población de metanógenos y protozoarios en el rumen (Tavendale et al., 2005), lo cual reduciría las emisiones de metano (Waghorn y Woodward, 2006).

Con base en estos antecedentes, el objetivo de esta investigación fue evaluar la cinética de la degradación ruminal de los nutrientes, digestibilidad aparente de los nutrientes, población de protozoarios del rumen, y producción de gas in vitro de dietas a base de biomasa pos-cosecha de Amaranthus cruentus.

\section{Materiales y Métodos}

\section{Localización}

El estudio se realizó en la Facultad de Ciencias Agropecuarias de la Universidad Técnica de Ambato, ubicada en el cantón Cevallos, provincia de Tungurahua, Ecuador, a una altitud de $2890 \mathrm{~m}$.

\section{Muestras Vegetales y Tratamientos}

Se trabajó con un cultivo de amaranto (A. cruentus) sembrada como fuente de grano para el consumo humano y una pastura de kikuyo (Pennisetum clandestinum).

La biomasa (planta) pos-cosecha de $A$. cruentus se cortó a $5 \mathrm{~cm}$ del suelo, inmediatamente después a la cosecha de la panoja ( 5 meses). Se colectó $30 \mathrm{~kg}$ de materia fresca que fue deshidratada exponiéndola al sol y procesada en un molino de martillo (CREMASCO DP2, Brasil) a un tamaño de partícula de $2 \mathrm{~mm}$. En forma similar, $30 \mathrm{~kg}$ de materia fresca de pasto fue cortado a una edad de 35 días y el proceso de secamiento fue similar al de A. cruentus.

Los forrajes molidos se incorporaron en una ración integral, de acuerdo a cuatro combinaciones (Cuadro 1).

La materia seca (MS) (\# 7.007) y ceniza (\# 7.009) se determinaron según la AOAC (1990). La fibra detergente neutro (FDA) y fibra detergente ácida (FDN) se determinaron mediante el método 12 y 13 respectivamente, ANKOM ${ }^{2000}$ analizador de fibra (ANKOM Technology, Macedon, NY, EEUU). La PC se determinó por análisis elemental (N) utilizando un LECO CHN 628 (LECO Corporation).

\section{Animales}

Se utilizaron seis toros con peso de 412.3 $\pm 35.2 \mathrm{~kg}$, provistos de una cánula ruminal (cuatro pulgadas de diámetro interno, Bar Diamond, Parma, Idaho, EEUU). Los animales se alojaron en corrales individuales y fueron alimentados con una dieta a base de Medicago sativa y P. clandestinum y se les proporcionó agua ad libitum.

\section{Degradación Ruminal in situ}

Se evaluó mediante la técnica de la bolsa de nylon en el rumen descrita por Ørskov 
Cuadro 1. Inclusión de niveles de rastrojo de Amaranthus cruentus y composición química de las dietas experimentales $(\mathrm{g} / \mathrm{kg} \mathrm{MS})$

\begin{tabular}{lcccc}
\hline \multirow{2}{*}{ Forrajes } & \multicolumn{4}{c}{ Tratamientos } \\
\cline { 2 - 5 } & $\mathrm{T} 1$ & $\mathrm{~T} 2$ & $\mathrm{~T} 3$ & $\mathrm{~T} 4$ \\
\hline P. clandestinum & & 1000 & 750 & 500 \\
A. cruentus & 1000 & & 250 & 500 \\
\hline Total & 1000 & 1000 & 1000 & 1000 \\
Composición química (\%) & & & & \\
Materia seca & 85.23 & 84.86 & 86.02 & 85.89 \\
Materia orgánica & 90.78 & 89.44 & 90.12 & 90.55 \\
Proteína cruda & 14.41 & 6.96 & 8.86 & 11.32 \\
Fibra detergente neutro & 58.30 & 66.52 & 66.04 & 61.84 \\
Fibra detergente ácida & 20.87 & 29.64 & 29.35 & 27.35 \\
Cenizas & 9.22 & 10.56 & 9.88 & 9.45 \\
\hline
\end{tabular}

et al. (1980). En cada animal se colocaron dos bolsas conteniendo $5 \mathrm{~g}$ MS de cada tratamiento y se incubaron por $0,4,8,12,24,36$, 48,72 y $96 \mathrm{~h}$. Al término de los periodos de incubación, las bolsas fueron removidas, lavadas con agua corriente y secadas a $60^{\circ} \mathrm{C}$. Las bolsas empleadas para medir la pérdida por lavado $(0 \mathrm{~h})$ no se incubaron en el rumen y solo se lavaron con agua corriente. Los residuos se almacenaron en bolsas de polietileno a $-4{ }^{\circ} \mathrm{C}$.

La desaparición de los nutrientes fue calculada como una proporción del material incubado y residual. Los datos se ajustaron a la ecuación: $\mathrm{Y}=\mathrm{a}+\mathrm{b}\left(1-\mathrm{e}^{-\mathrm{ct}}\right)$ y la degradación efectiva se ajustó mediante la ecuación $\mathrm{DE}=\mathrm{a}+[(\mathrm{b} * \mathrm{c}) /(\mathrm{c}+\mathrm{k})]$ considerando una tasa de pasaje de 5\% (Ørskov y McDonald, 1979).

\section{Digestibilidad Aparente, Población de Protozoarios Ruminales y Producción de Gas}

Estas pruebas se realizaron in vitro. Para esto, se obtuvo el contenido del rumen- (líquido y fracciones sólidas) por separado de cada toro canulado. El contenido ruminal $(1000 \mathrm{ml})$ se recolectó antes de proporcionar el alimento y se mantuvo a $39^{\circ} \mathrm{C}$ en un recipiente de plástico sellado durante el transporte al laboratorio.

El análisis en el laboratorio se hizo dentro de la hora de la recolección en un medio rico en nitrógeno según Menke y Steingass (1988). La producción de gas se determinó según la metodología descrita por Theodorou et al. (1994). En breve, $0.5 \mathrm{~g}$ de MS de cada tratamiento se colocó en botellas de vidrio de $100 \mathrm{ml}$ de capacidad nominal, se añadió 60 $\mathrm{ml}$ de inóculo ruminal (70:30 medio/inóculo ruminal) bajo un flujo de $\mathrm{CO}_{2}$ constante. Las botellas se sellaron y se incubaron a $39-40{ }^{\circ} \mathrm{C}$. La presión de gas y el volumen se midieron manualmente a las $3,6,9,12,18,24,36$ y 48 $\mathrm{h}$ después de la incubación con un transductor de presión DELTA OHM modelo DO 9704 (Delta OHM, Padova, Italia) y jeringas plásticas. Para cada tratamiento se utilizaron seis botellas (repeticiones) por cada tiempo de incubación y cuatro botellas adicionales como blanco. 
Al final de la incubación, la digestibilidad in vitro (MS fermentable, materia orgánica [MO] y PC) se estimó mediante el filtrado de los residuos y se corregió con la MS y la PC residual de los frascos utilizados como blancos. Se estimó la producción total de gas por $0.5 \mathrm{~g}$ MS fermentable, así como la digestibilidad aparente de MS, MO y PC.

Se utilizaron seis botellas adicionales por tiempo de incubación $(0,24,36$ y 48 h) para determinar la población de protozoarios del rumen antes y después de exponerlos a las dietas integrales. Al término de cada periodo de incubación se retiró de cada botella $1 \mathrm{ml}$. Los protozoarios se conservaron con una gota de formol y se mantuvo a $4{ }^{\circ} \mathrm{C}$ hasta cuantificarlos utilizando un microscopio óptico (x40) y una cámara Fucsh-Rosenthal. Los protozoarios se tiñeron con una solución de verde de metilo formamida según la metodología descrita por Ogimoto y Imai (1981).

\section{Diseño Experimental y Análisis Estadís- tico}

Se utilizó un diseño completamente al azar con 4 tratamientos y 6 repeticiones. Los resultados se sometieron a un análisis de varianza mediante el PROC GLM del SAS (SAS, 2002). Las medias se evaluaron usando la prueba de Tukey, a excepción de la cinética de degradación ruminal in situ de los nutrientes, la cual se analizó con el programa Graphpad Prism 6 (San Diego, EEUU).

\section{Resultados}

Los parámetros de la cinética de degradación ruminal de los nutrientes muestran diferencias $(p=0.0001)$ entre tratamientos, siendo T1 el tratamiento que obtuvo la mayor degradación de MS, PC y FDN (solo $A$. cruentus), tanto para la fracción soluble (a), la fracción insoluble pero potencialmente degradable (b), la tasa de degradación en porcentaje por hora (c) y la degradación efectiva (DE) (Cuadro 2).
La digestibilidad in vitro de la MS, MO y PC mostró diferencias significativas $(p<0.05)$ entre tratamientos. La mayor digestibilidad fue para los tratamientos $\mathrm{T} 1$ : solo A. cruentus $(60,68$ y 59\%, respectivamente) y T4: 50:50 A. cruentus y $P$. clandestinum (53, 61 y 51\%, respectivamente) (Cuadro 3 ). La menor producción de gas in vitro fue para $\mathrm{T} 1$, con una diferencia de $91 \mathrm{ml} / 0.5 \mathrm{~g}$ MSF con respecto a los tratamientos de mayor producción de gas (T2 y T3) (Cuadro 3; $\mathrm{p}=0.0001$ ).

La población de protozoarios Holotricos en $\mathrm{T} 1$ a las 24 de incubación in vitro fue menor que en los demás tratamientos $(\mathrm{p}=0.0081)$. En el caso de los protozoarios Entodiniomorfos no se observaron diferencias numéricas entre tratamientos (Cuadro 4; $\mathrm{p}=0.0596$ ). Sin embargo, a partir de las 36 $\mathrm{h}$ se redujo a cero el número de Holotricos en los tratamientos T1, T3 y T4, y menor número de Entodiniomorfos en $\mathrm{T} 1$ con respecto a los demás tratamientos (Cuadro 4; $\mathrm{p}<0.05$ ).

\section{Discusión}

La mayor degradación ruminal y tasa de degradación en porcentaje por hora (c) de los nutrientes en el T1 y T4 (Cuadro 2), fue debido al menor contenido de carbohidratos estructurales que tiene la biomasa pos cosecha de A. cruentus (Cuadro 1). El menor contenido de carbohidratos propicia mayor acceso a los microrganismos ruminales y facilita su degradación (Salem et al., 2011; Peng et al., 2014). Por otro lado, la pared celular en T2 y T3 presenta mayor lignificación y, por ende, dificulta la acción de los microorganismos. Esto se observa claramente en este estudio, donde al incorporar $A$. cruentus en la ración aumenta la degradación efectiva de la fibra y proteína (Cuadro 2); resultados que coinciden con los reportados por Torres et al. (2013) y por BarrosRodriguez et al. (2015). 
Cuadro 2. Cinética de degradación ruminal in situ de los nutrientes de dietas a base de rastrojo de Amaranthus cruentus (g/kgMS)

\begin{tabular}{|c|c|c|c|c|c|c|}
\hline & \multicolumn{4}{|c|}{ Tratamientos } & \multirow{2}{*}{ EEM } & \multirow{2}{*}{ Valor $\mathrm{P}$} \\
\hline & $\mathrm{T} 1$ & $\mathrm{~T} 2$ & $\mathrm{~T} 3$ & $\mathrm{~T} 4$ & & \\
\hline \multicolumn{7}{|c|}{ Degradación de la MS } \\
\hline$T_{0}$ & 224.9 & 228.4 & 219.9 & 221.6 & & \\
\hline $\mathrm{a}$ & $200.9^{\mathrm{a}}$ & $189.2^{\mathrm{b}}$ & $190.1^{\mathrm{b}}$ & $193.8^{\mathrm{b}}$ & 8.34 & 0.0001 \\
\hline $\mathrm{b}$ & $428.8^{\mathrm{a}}$ & $360.6^{\mathrm{b}}$ & $372.9^{\mathrm{b}}$ & $376.1^{\mathrm{b}}$ & 10.32 & 0.0001 \\
\hline $\mathrm{c}$ & $0.054^{\mathrm{a}}$ & $0.02^{\mathrm{b}}$ & $0.018^{\mathrm{b}}$ & $0.028^{\mathrm{b}}$ & 0.0045 & 0.0001 \\
\hline $\mathrm{DE}$ & $423.5^{\mathrm{a}}$ & $292.2^{\mathrm{b}}$ & $288.8^{\mathrm{b}}$ & $328.8^{\mathrm{b}}$ & 11.06 & 0.0001 \\
\hline \multicolumn{7}{|c|}{ Degradación de la PC } \\
\hline$T_{0}$ & 182.3 & 180.1 & 172.7 & 179.5 & & \\
\hline $\mathrm{a}$ & $197.4^{\mathrm{a}}$ & $177.5 \mathrm{c}$ & $179.2^{\mathrm{bc}}$ & $182.9^{\mathrm{b}}$ & 5.23 & 0.0021 \\
\hline b & $376.2^{\mathrm{a}}$ & $300.3^{\mathrm{b}}$ & $312.6^{\mathrm{b}}$ & $319.8^{b}$ & 8.54 & 0.0001 \\
\hline $\mathrm{c}$ & $0.061^{\mathrm{a}}$ & $0.023^{\mathrm{b}}$ & $0.02^{\mathrm{b}}$ & $0.023^{\mathrm{b}}$ & 0.0078 & 0.0001 \\
\hline $\mathrm{DE}$ & $404.1^{\mathrm{a}}$ & $272.1^{\mathrm{b}}$ & $268.5^{\mathrm{b}}$ & $283.7^{\mathrm{b}}$ & 7.43 & 0.0001 \\
\hline \multicolumn{7}{|c|}{ Degradación de la FDN } \\
\hline$T_{0}$ & 162.3 & 159.0 & 161.7 & 170.2 & & \\
\hline $\mathrm{a}$ & $202.4^{\mathrm{a}}$ & $168.4^{\mathrm{b}}$ & $169.3^{b}$ & $171.7^{\mathrm{b}}$ & 10.54 & 0.0001 \\
\hline $\mathrm{b}$ & $310.6^{\mathrm{a}}$ & $279.8^{\mathrm{b}}$ & $287.2^{\mathrm{b}}$ & $289.0^{\mathrm{b}}$ & 7.65 & 0.0001 \\
\hline c & $0.048^{\mathrm{a}}$ & $0.02^{\mathrm{b}}$ & $0.021^{\mathrm{b}}$ & $0.023^{\mathrm{b}}$ & 0.0081 & 0.0023 \\
\hline $\mathrm{DE}$ & $354.5^{\mathrm{a}}$ & $248.3^{\mathrm{c}}$ & $254.2^{\mathrm{bc}}$ & $262.8^{\mathrm{b}}$ & 9.76 & 0.0001 \\
\hline
\end{tabular}

abc Medias con letras distintas entre filas difieren significativamente $(p<0.05)$

$T_{0}$ : tiempo cero (muestras lavadas en laboratorio); a: fracción soluble; b: fracción insoluble pero potencialmente degradable; c: tasa de degradación en \% por hora; DE: degradación efectiva; EEM: error estándar de la media

T1: rastrojo de amaranto; T2: forraje de $P$. clandestinum; T3: $P$. clandestinum $75 \%+$ rastrojo de $A$. cruentus $25 \%$; T4: P. clandestinum $50 \%$ + rastrojo de $A$. cruentus $50 \%$

La mayor digestibilidad in vitro de los nutrientes en T1 y T4 fue debido al menor contenido de FDN y mayor PC que aporta A. cruentus a la ración (Cuadro 1), ya que la degradación de las proteínas da como resultado péptidos, $\mathrm{NH}_{3}$, aminoácidos y ácidos grasos de cadena corta ramificados; compuestos que pueden mejorar las funciones del rumen. Esto estimula el crecimiento microbiano del rumen en general y, específicamente, de las bacterias celulolíticas y proteolíticas, trayendo como consecuencia una mayor digestión de los nutrientes (Hoover y Stokes, 1991); aspecto parcialmente obser- vado en esta investigación (Cuadro 3). Estos resultados concuerdan con los reportados por Barros-Rodríguez et al. (2014).

La menor producción de gas in vitro en T1 y T4 (Cuadro 2), consistente con los resultados de Chai et al. (2004) fue debido posiblemente a: a) el bajo contenido de FDN en las raciones con biomasa poscosecha de $A$. cruentus; b) una mejor utilización del nitrógeno y mayor síntesis de proteína microbial (Blummel et al., 1997; Elghandour et al., 2017); y c) una reducción de las poblaciones de protozoarios (Cuadro 4) y posible dismi- 
Cuadro 3. Digestibilidad in vitro (DIV) de los nutrientes (Materia seca [MS], materia orgánica [MO] y proteína cruda [PC]) y producción de gas in vitro de dietas a base de rastrojo de amaranto

\begin{tabular}{|c|c|c|c|c|c|c|}
\hline & \multicolumn{4}{|c|}{ Tratamientos } & \multirow{2}{*}{ EEM } & \multirow{2}{*}{ Valor $\mathrm{F}$} \\
\hline & $\mathrm{T} 1$ & $\mathrm{~T} 2$ & T3 & $\mathrm{T} 4$ & & \\
\hline DIVMS g/kg & $600.4^{\mathrm{a}}$ & $500.9^{b}$ & $442.8^{c}$ & $531.3^{\mathrm{ab}}$ & 27.78 & 0.0060 \\
\hline DIVMO g/kg & $682.7^{\mathrm{a}}$ & $576.2^{\mathrm{b}}$ & $552.1^{\mathrm{b}}$ & $618.8^{\mathrm{ab}}$ & 21.01 & 0.0002 \\
\hline DIVPC g/kg & $592.7^{\mathrm{a}}$ & $480.5^{\mathrm{b}}$ & $494.6^{\mathrm{b}}$ & $518.3^{\mathrm{ab}}$ & 25.76 & 0.0001 \\
\hline PGIV ml/0.5 g MSF & $234.6^{\mathrm{c}}$ & $334.7^{\mathrm{a}}$ & $325.9^{\mathrm{a}}$ & $280.5^{\mathrm{b}}$ & 7.34 & 0.0001 \\
\hline
\end{tabular}

abc Medias con letras distintas entre filas difieren significativamente $(p<0.05)$

EEM: error estándar de la media; PGIV: producción de gas in vitro; MSF: materia seca fermentable T1: rastrojo de Amaranthus cruentus; T2: forraje de P. clandestinum; T3: P. clandestinum $75 \%+$ rastrojo de $A$. cruentus 25\%; T4: $P$. clandestinum $50 \%+$ rastrojo de $A$. cruentus $50 \%$

Cuadro 4. Poblaciones de protozoarios ruminales in vitro $\left(\log _{10}\right)$ antes y después de exponerlos a dietas a base de rastrojo de $A$. cruentus

\begin{tabular}{|c|c|c|c|c|c|c|}
\hline \multirow{2}{*}{ Protozoos } & \multicolumn{4}{|c|}{ Tratamientos } & \multirow{2}{*}{ EEM } & \multirow{2}{*}{ Valor $P$} \\
\hline & $\mathrm{T} 1$ & $\mathrm{~T} 2$ & $\mathrm{~T} 3$ & $\mathrm{~T} 4$ & & \\
\hline \multicolumn{7}{|l|}{ Hora 0} \\
\hline $\mathrm{H}$ & $4.02^{\mathrm{a}}$ & $3.90^{\mathrm{a}}$ & $4.04^{\mathrm{a}}$ & $3.63^{\mathrm{a}}$ & 0.111 & 0.0588 \\
\hline E & $4.75^{\mathrm{a}}$ & $4.62^{\mathrm{a}}$ & $4.84^{\mathrm{a}}$ & $4.74^{\mathrm{a}}$ & 0.058 & 0.1142 \\
\hline \multicolumn{7}{|l|}{ Hora 24} \\
\hline $\mathrm{H}$ & $0.56^{\mathrm{c}}$ & $2.98^{\mathrm{a}}$ & $2.46^{\mathrm{a}}$ & $1.26^{\mathrm{b}}$ & 0.696 & 0.0081 \\
\hline E & $4.64^{\mathrm{a}}$ & $4.29^{\mathrm{a}}$ & $4.51^{\mathrm{a}}$ & $4.37^{\mathrm{a}}$ & 0.074 & 0.0596 \\
\hline \multicolumn{7}{|l|}{ Hora 36} \\
\hline $\mathrm{H}$ & $0^{\mathrm{b}}$ & $0.56^{\mathrm{a}}$ & $0^{\mathrm{b}}$ & $0^{\mathrm{b}}$ & 0.283 & 0.0413 \\
\hline E & $1.70^{\mathrm{b}}$ & $3.81^{\mathrm{a}}$ & $3.71^{\mathrm{a}}$ & $3.26^{\mathrm{a}}$ & 0.510 & 0.0295 \\
\hline \multicolumn{7}{|l|}{ Hora 48} \\
\hline $\mathrm{H}$ & $0^{\mathrm{b}}$ & $0.78^{\mathrm{a}}$ & $0^{\mathrm{b}}$ & $0^{\mathrm{b}}$ & 0.132 & 0.0098 \\
\hline E & $1.80^{\mathrm{b}}$ & $4.14^{\mathrm{a}}$ & $3.89^{\mathrm{a}}$ & $3.86^{\mathrm{a}}$ & 0.420 & 0.0026 \\
\hline
\end{tabular}

${ }^{a b c}$ Medias con letras distintas entre filas difieren significativamente $(p<0.05)$

$\mathrm{H}$ : Holotricos; E: Entodiniomorfos; EEM: error estándar de la media

T1: rastrojo de $A$. cruentus; T2: forraje de $P$. clandestinum; T3: $P$. clandestinum $75 \%+$ rastrojo de $A$. cruentus $25 \%$; T4: $P$. clandestinum $50 \%+$ rastrojo de $A$. cruentus $50 \%$ 
nución de los microorganismos metanogénicos (Jayanegara et al., 2012), ante la acción de los compuestos secundarios presentes en la ración (Nana et al., 2012).

La disminución in vitro de las poblaciones de protozoarios Holotricos y Entodiniomorfos observado en los tratamientos con A. cruentus (Cuadro 4), fue debido posiblemente a la acción directa de los compuestos secundarios (taninos y saponinas) presentes en la biomasa pos-cosecha de $A$. cruentus (Nana et al., 2012). Estos compuestos tienen un efecto defaunante en el rumen; por ejemplo, el efecto selectivo de las saponinas hacia el colesterol de la membrana celular de los protozoarios que causa su lisis (Jayanegara et al., 2012). Estos resultados son similares a los reportados por Monforte-Briceño et al. (2005), González et al. (2011) y BarrosRodríguez et al. (2015), quienes reportan un efecto defaunante atribuidos a los taninos y saponinas presentes en forrajes de plantas arbóreas y arbustivas.

\section{Conclusión}

La mezcla de la biomasa pos-cosecha de Amaranthus cruentus con forrajes de baja calidad nutriticional puede incrementar la degradación y digestibilidad de los nutrientes, reducir la producción de gases de efecto invernadero y las poblaciones de protozoarios del rumen, lo cual podría maximizar la productividad de los rumiantes en condiciones in vivo.

\section{Literatura Citada}

1. Abdulrazak SA, Fujihara T, Ondiek JK, Orskov ER. 2000. Nutritive evaluation of some Acacia tree leaves from Kenya. Anim Feed Sci Technol 85: 89-98. doi: 10.1016/S0377-8401(00)$00133-4$
2. [AOAC] Association of Official Analytical Chemists. 1990. Official methods of analysis. $15^{\text {th }}$ ed. Arlington, VA, USA: AOAC. 684 p.

3. Barros-Rodríguez MA, SolorioSánchez FJ, Sandoval-Castro CA, Ahmed AMM, Rojas-Herrera $R$, Briceño-Poot EG, Ku-Vera JC. 2014. Effect of intake of diets containing tannins and saponins on in vitro gas production and sheep performance. Anim Prod Sci 54: 1486-1489. doi: 10.1071/ AN14294

4. Barros-Rodríguez MA, SolorioSánchez FJ, Sandoval-Castro CA, Klieve A, Rojas-Herrera RA, BriceñoPoot EG, Ku-Vera JC. 2015. Rumen function in vivo and in vitro in sheep fed Leucaena leucocephala. Trop Anim Health Prod 47: 757-764. doi: 10.1007/ s11250-015-0790-y

5. Blummel M, Makkar HPS, Becker K. 1997. In vitro gas production: a technique revisited. J Anim Physiol Anim Nutr 77: 24-34. doi: 10.1111/j.14390396.1997.tb00734.x

6. Chai WZ, van Gelder AH, Cone JW. 2004. Relationship between gas production and starch degradation in feed samples. Anim Feed Sci Technol 114: 195-204. doi: 10.1016/j.anifeedsci.2003.11.014

7. Elghandour MMY, Vázquez JC, Salem AZM, Kholif AE, Cipriano MM, Camacho LM, Márquez O. 2017. In vitro gas and methane production of two mixed rations influenced by three different cultures of Saccharomyces cerevisiae. J Appl Anim Res 45: 389395. doi: 10.1080/09712119.2016.1204304

8. González N, Galindo J, Aldana AI, Moreira O, Sarduy L. 2011. Effect of four mulberry (Morus alba Linn) varieties on microbial population and fermentative products with rumen liquid from river buffaloes (Bubalus bubalis) under in vitro conditions. Cuban J Agr Sci 45: 399-404. 
9. García-Pereyra J, Valdés-Lozano CGS, Olivares-Saenz E, AlvaradoGómez O, Alejandre Iturbide G, Salazar-Sosa E, Medrano-Roldán H. 2009. Rendimiento de grano y calidad del forraje de amaranto (Amaranthus spp) cultivado a diferentes densidades en el noreste de México. Phyton (Buenos Aires) 78: 53-60.

10. Hoover WH, Stokes SR. 1991. Balancing carbohydrates and proteins for optimum rumen microbial yield. J Dairy Sci 74: 3630-3644. doi: 10.3168/ jds.S0022-0302(91)78553-6

11. Jayanegara A, Leiber F, Kreuzer M. 2012. Meta-analysis of the relationship between dietary tannin level and methane formation in ruminants from in vivo and in vitro experiments. J Anim Physiol Anim Nutr 96: 365-375. doi: 10.1111/j.1439-0396.2011.01172.x

12. Kurihara M, Magner T, Hunter RA, McCrabb GJ. 1999. Methane production and energy partition of cattle in the tropics. Br J Nutr 81: 227-234. doi: $10.1017 / \mathrm{S} 0007114599000422$

13. Menke KH, Steingass H. 1988. Estimation of the energetic feed value obtained from chemical analysis and in vitro gas production using rumen fluid. Anim Res Develop 28: 7-55.

14. Monforte-Briceño GE, Sandoval-Castro CA, Ramírez-Avilés L, CapetilloLeal CM. 2005. Defaunating capacity of tropical fodder trees: effects of polyethylene glycol and its relationship to in vitro gas production. Anim Feed Sci Technol 123: 313-327. doi: 10.1016/ j.anifeedsci.2005.04.016

15. Manotoa S. 2016. Capacidad de defaunación ruminal y mitigación de gases de efecto invernadero: efecto de leguminosas forrajeras arbóreas y arbustivas. Tesis de Magíster. Ambato: Univ Técnica de Ambato. $48 \mathrm{p}$.

16. Nana FW, Hilou A, Millogo JF, Nacoulma OG. 2012. Phytochemical composition, antioxidant and xanthine oxidase inhibitory activities of Amaranthus cruentus L and Amaranthus hybridus L extracts. Pharmaceuticals 5: 613-628. doi: $10.3390 / \mathrm{ph} 5060613$

17. Ogimoto K, Imai S. 1981. Atlas of rumen microbiology. Japan: Scientific Societies Press. $231 \mathrm{p}$.

18. Orskov ER, Hovell FDB, Mould F. 1980. The use of the nylon bag technique for the evaluation of feedstuffs. Trop Anim Prod 5: 195-213.

19. Orskov E, McDonald I. 1979. The estimation of protein degradability in the rumen from incubation measurements weighted according to rate of passage. J Agric Sci 92: 499-503. doi: 10.1017/ S0021859600063048

20. Peng $Q$, Khan NA, Wang Z, Yu P. 2014. Relationship of feeds protein structural makeup in common prairie feeds with protein solubility, in situ ruminal degradation and intestinal digestibility. Anim Feed Sci Technol 194: 58-70. doi: 10.1016/j.anifeedsci.2014.05.004

21. Salem AFZ, El-Adawy M, Gado H, Camacho LM, Ronquillo M, Alsersy H, Borhami BE. 2011. Effects of exogenous enzymes on nutrients digestibility and growth performance in sheep and goats. Trop Subtrop Agroecosyst 14: 867-874.

22. SAS. 2002. Institute Inc., SAS/STAT. Software v. 9.00. Cary, NC, USA.

23. Tan HY, Sieo CC, Abdullah N, Liang $J B$, Huang XD, Ho YW. 2011. Effects of condensed tannins from Leucaena on methane production, rumen fermentation and populations of methanogens and protozoa in vitro. Anim Feed Sci Technol 169: 185-193. doi: 10.1016/j.anifeedsci.2011.07.004

24. Tavendale MH, Lane GA, Schreurs NM, Fraser K, Meagher LP. 2005. The effects of condensed tannins from Dorycnium rectum on skatole and indole ruminal biogenesis for grazing sheep. Aust J Agric Res 56: 1331-1337. doi: 10.1071/AR04232 
25. Theodorou M, Williams B, Dhanoa M, Mcallan A, France J. 1994. A simple gas production method using a pressure transducer to determine the fermentation kinetics of ruminant feeds. Anim Feed Sci Technol 48: 185-197. doi: 10.1016/ 0377-8401(94)90171-6

26. Torres N, Mendoza GD, Bárcena JR, González SS, Loera O, Salem AZM, Lara A. 2013. Effect of a fibrolytic enzymatic extract from Cellulomonas flavigena on in vitro degradation and in vivo digestibility and productive performance of lambs. Anim Feed Sci Technol 13: 583-592.

27. Waghorn GC, Woodward SL. 2006. Ruminant contributions to methane and global warming - a New Zealand perspective. In: Bhatti JS, Lal R, Apps MJ, Price MA (eds). Climate change and managed ecosystems. Boca Raton, Fl, USA: CRC Press. p 233-260. 